\title{
Cyclic and Explosive Evaluation of New Proposed Steel Joint
}

\author{
Iman Faridmehr, ${ }^{1}$ Yusof Ahmad, ${ }^{2}$ Mahmood Md. Tahir, ${ }^{1}$ and Mohd Hanim Osman ${ }^{1}$ \\ ${ }^{1}$ UTM Construction Research Centre (CRC), Institute of Smart Infrastructures and Innovative Construction, \\ Universiti Teknologi Malaysia (UTM), 81300 Skudai, Johor Bahru, Malaysia \\ ${ }^{2}$ Faculty of Civil Engineering, Universiti Teknologi Malaysia, 81310 Johor, Malaysia
}

Correspondence should be addressed to Mahmood Md. Tahir; mahmoodtahir@utm.my

Received 2 August 2015; Revised 23 October 2015; Accepted 1 November 2015

Academic Editor: John Mander

Copyright (C) 2016 Iman Faridmehr et al. This is an open access article distributed under the Creative Commons Attribution License, which permits unrestricted use, distribution, and reproduction in any medium, provided the original work is properly cited.

\begin{abstract}
The behaviour of a novel steel beam-to-column connection, the saddlebag, subjected to cyclic and progressive collapse, was evaluated in this paper. The cyclic behaviour considered the interstory drift angle and flexural strength in accordance with 2010 AISC Seismic Provisions, while progressive collapse assessment was evaluated through the plastic hinge rotation angle based on acceptance criteria provided in the UFC 4-023-03 guideline. From the cyclic test, one complete cycle of an interstory drift angle of $0.06 \mathrm{rad}$ was satisfied for the saddlebag connection, which is an indication of the effectiveness in accordance with 2010 AISC Seismic Provisions. Besides, the new proposed connection developed adequate catenary action, which is a fundamental criterion to resist against progressive collapse. The resulting fuller hysteretic loops with large energy dissipation capacity in the proposed saddlebag connection guarantee its ability to address the inelastic deformation demands in earthquake conditions.
\end{abstract}

\section{Introduction}

After the 1994 Northridge, California, earthquake, given the damage to steel moment frame connections, there was great concern among engineers regarding the authenticity of the established construction and design procedures. Damage occurred in beam-to-column joints experiencing rotation levels way below the yield capacity of the framing members. This unexpected brittle fracture was contrary to the projected design philosophy and performance of these frames, which was the dissipation of energy through the formation of ductile plastic hinges in steel beams. After the earthquake, a great deal of research was conducted to investigate the behaviour of fully restrained connections [1-5]. It was generally concluded that adequate inelastic rotational capacity of sufficient reliability cannot be provided using the pre-Northridge connections, where fracture led to failure of all connections at or close to the beam flange groove welds. Figure 1(a) illustrates a sample detail of a tested connection, and Figure 1(b) shows its experimental response, having plotted the bending moment at the column face against plastic rotation. Similar to many other pre-Northridge specimens, no plastic moment of the beam was developed by this connection and a premature failure with mainly no ductility was exhibited by this connection as well.

Meanwhile, since the 1968 Ronan Point apartment tower disaster, which led to the partial collapse of the building, the significance of structural resistance to progressive collapse had become more evident. Furthermore, this accelerated trend of investigation was redoubled after the September 11 attacks. After the occurrence of high profile structural failures due to progressive collapse, the previous research on this subject continued at a faster rate [6-10]. This research revealed that, by the time an interior column is removed from the structure by an explosion or a terrorist attack, for instance, an alternative load path will be developed by the adjacent structural assemblage, including beams, columns, and joints. Besides, it was found that the extent of catenary action was controlled by the rotational capacity of beam-tocolumn joints. Figure 2 shows how, following the elimination of a structural support, high catenary forces resulting from subsequent large deformations will be produced in the system. Accordingly, both the beam-to-column connection and the beam cross section must resist the huge axial forces that are created while undergoing large deformations. 


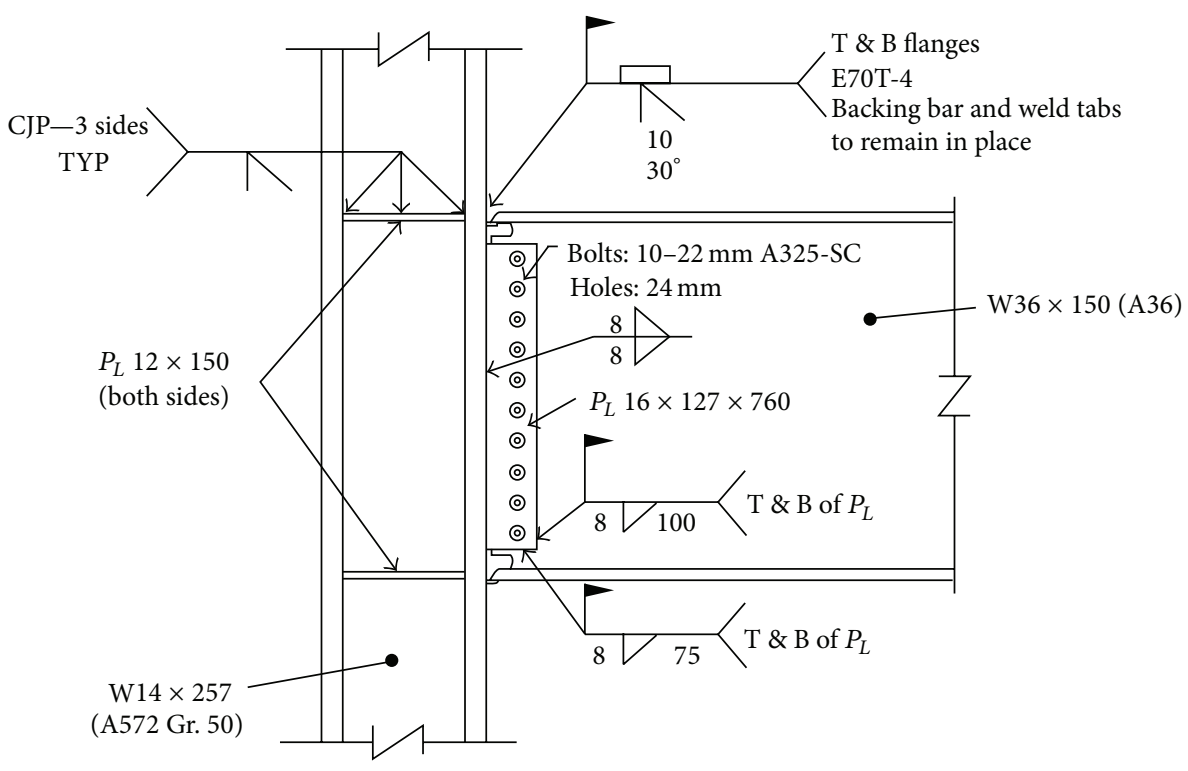

(a) Connection detail for test specimen

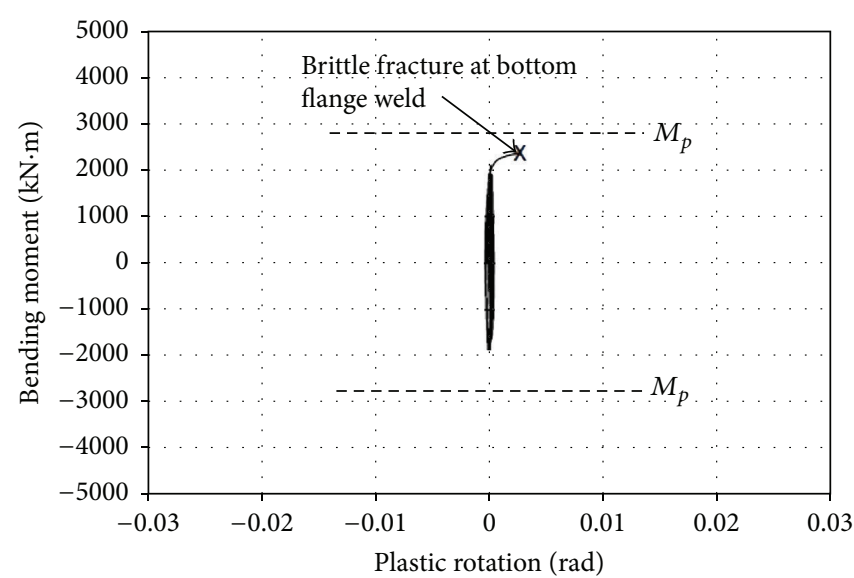

(b) Response of test specimen

FIGURE 1: Example of experimental response of pre-Northridge connections.

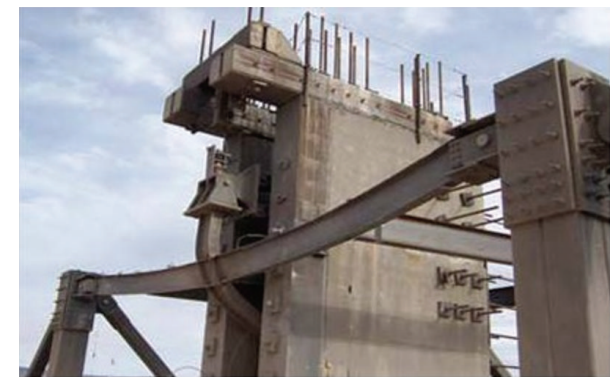

(a)

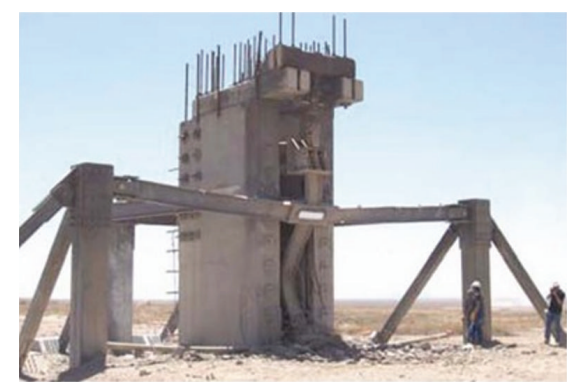

(b)

FIGURE 2: Postblast images of a standard moment connection (a) and a side-plate reinforced blast resistant connection (b) [7]. 


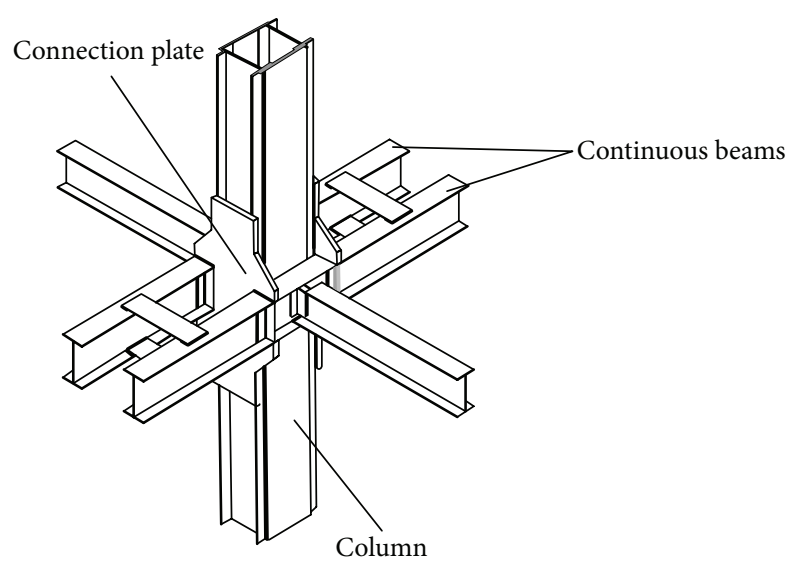

FIGURE 3: The proposed saddlebag connection.

Nowadays, structural engineering communities are considering the following two major strategies to address extreme loading requirements for steel beam-to-column connections:

(i) Strengthening strategy: such elements as cover plates, haunches, side plate, and vertical ribs are utilized to upgrade or enhance the load carrying capacity of the connection.

(ii) Weakening strategy: the most preeminent example of this strategy is Reduced Beam Section or RBS connection, where a beam element (usually flange) is intentionally weakened in bending (by reducing the width of the flanges) to create a "fuse" for ductile yield mechanism without connection failure.

The main strategy considered in the proposed saddlebag connection was to improve the catenary action as a major factor to resist progressive collapse. Two parallel beams positioned on both sides of a column are responsible for the provision of adequate catenary action (Figure 3). Provision of a clearer and simpler load transfer regime by means of this connection reduces the complexity of the stress distribution and also simplifies the complicated calculations of the peak stress. Besides, in the case of a terrorist attack, the continuity of beams and columns will provide a superior ability for the connection assembly to tolerate unexpected downside column losses; hence, the frame will be protected against progressive collapse. The connection of the column to the beams is provided through the application of a connection plate that will be welded to the column flanges. There is no need to consider the lateral resistant system in a direction with continuous beams as it is believed that the proposed connection can address the seismic requirements. However, in the orthogonal direction the lateral resistant system should be provided. The unique configuration of the saddlebag connection was considered to ensure that all major energy dissipations are created outside the column's shear panel zone.

This research involves two individual specimens which are evaluated by numerical simulation of the process of cyclic and push-down loading. For seismic assessment, the interstory drift angle and flexural strength in accordance with
2010 AISC Seismic Provisions [12] were taken into account; while to evaluate the progressive collapse behaviour of the connection, rotational capacities in conformity with 2010 UFC 4-023-03 [13] guideline were considered.

\section{Design Procedures of Saddlebag Connection}

The lateral loading was considered to design saddlebag connection plate. Figure 4 shows the yielding mechanism and load transfer to connection plate in case of lateral loading. The vertical load of one of the beams, resulting from the combination of gravity and lateral loads, exerted on the right side connection plate is determined from the following formula:

$$
F=\frac{M_{p b 1}+M_{p b 2}}{d_{1}}+V_{g 1},
$$

where $M_{p b 1}$ and $M_{p b 2}$ are the expected flexural strengths of the beams at the left and right side of connection, respectively. $V_{g 1}$ is the shear force from one of the beams at right side of connection due to gravity load. $d_{1}$ is horizontal distance between two connection plates.

According to Figure 5, the minimum length to provide the sufficient contact between the connection plate and beam flange, $L_{1}$, is $b_{f b}+0.5 \mathrm{~mm}$, where $b_{f b}$ is defined as the beam flange width. The minimum length to provide the sufficient space for welding between the connection plate and column flange, $L_{2}$, is $b_{f c}+3 \mathrm{~mm}$, where $b_{f c}$ is defined as the column flange width. Since fabrication of the connection plate is done in the shop, the length of $L_{3}$ is taken as the beam depth, $d_{b}$. Furthermore, the minimum length of $L_{4}$ to transmit the vertical force, $F$, from the fillet weld to the column is determined from the following formula:

$$
L_{4}=\frac{F}{R_{w}^{\prime}}
$$

where $R_{w}^{\prime}$ is the weld strength per unit length during extreme loading [14].

The philosophy behind considering the haunch for the saddlebag connection plate is to provide high moment transfer from the connection plate to the column during a seismic event. The haunch will reduce the connection plate thickness, which is calculated in accordance with plate buckling theory. Accordingly, it is recommended that the minimum lengths of $L_{5}$ and $L_{6}$ be taken as $1.5 L_{1}$ and $0.4 L_{5}$. To design the connection plate thickness, plate and shell buckling theory was employed in accordance with Timoshenko et al. [11] as follows:

$$
\sigma_{\mathrm{cr}}=\frac{k \pi^{2} E}{12\left(1-v^{2}\right)(b / t)^{2}},
$$

where $k$ is the plate buckling coefficient. The buckling coefficient, $k$, is a function of the support condition along the longitudinal edges and the type of loading. To ensure that connection plate fails by yielding rather than buckling, the corresponding critical buckling stress should be greater than the yield stress. Equating the expression given in (3) to the 


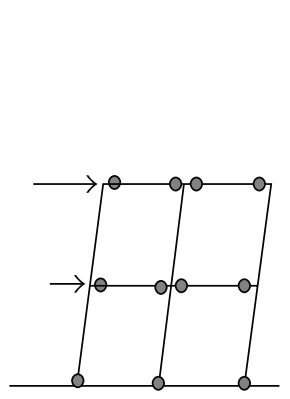

(a)

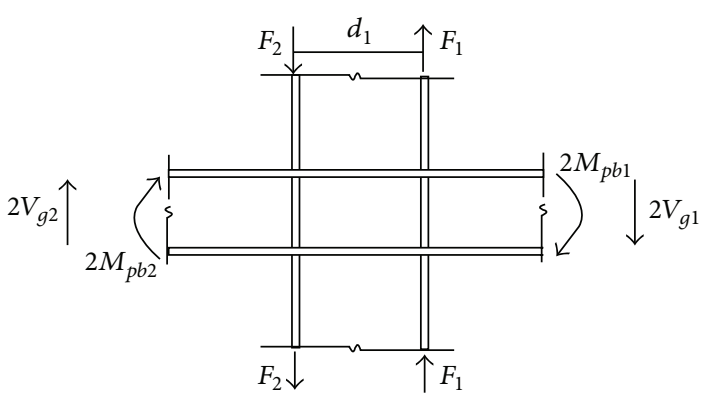

(b)

FIGURE 4: Load transfer path of saddlebag connection: (a) yielding mechanism and (b) vertical load transfer to the connection plates.

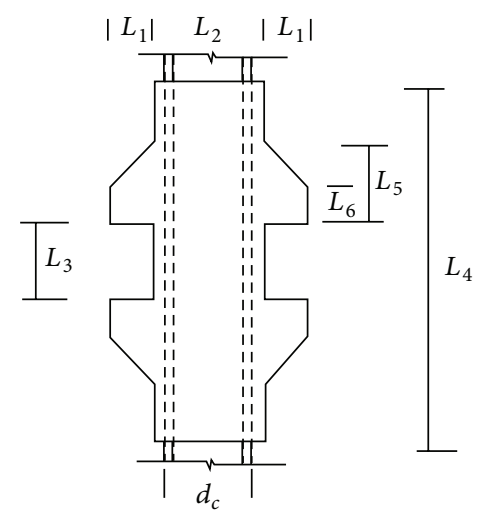

Figure 5: Connection plate dimensions.

yield stress, the limiting value of the width-thickness ratio to ensure yielding before plate buckling can be obtained as [14]

$$
\frac{b}{t} \leq\left\{\frac{k \pi^{2} E}{12\left(1-v^{2}\right)\left(f_{y}\right)}\right\}^{1 / 2} .
$$

The $k$ values for various common support conditions and loading cases are illustrated in Figure 6. Notice that values given are based on plates having loaded edges simply supported.

The connection plate weld to the beam top and bottom flanges to avoid any movement during extreme loading events. Accordingly, the connection plate subjected to the bending stress as well as compression stress is shown in Figure 7.

The amount of applied loads is determined from the following formula:

$$
\begin{aligned}
& F_{1}=F_{2}=\frac{2 M}{d_{c}}, \\
& F_{3}=F_{4}=\frac{M}{d_{b}},
\end{aligned}
$$

where $M$ is the expected flexural strengths of the beams at the left and right side of connection. $d_{c}$ is horizontal distance between two connection plates. $d_{b}$ is horizontal distance between two flanges of beam.

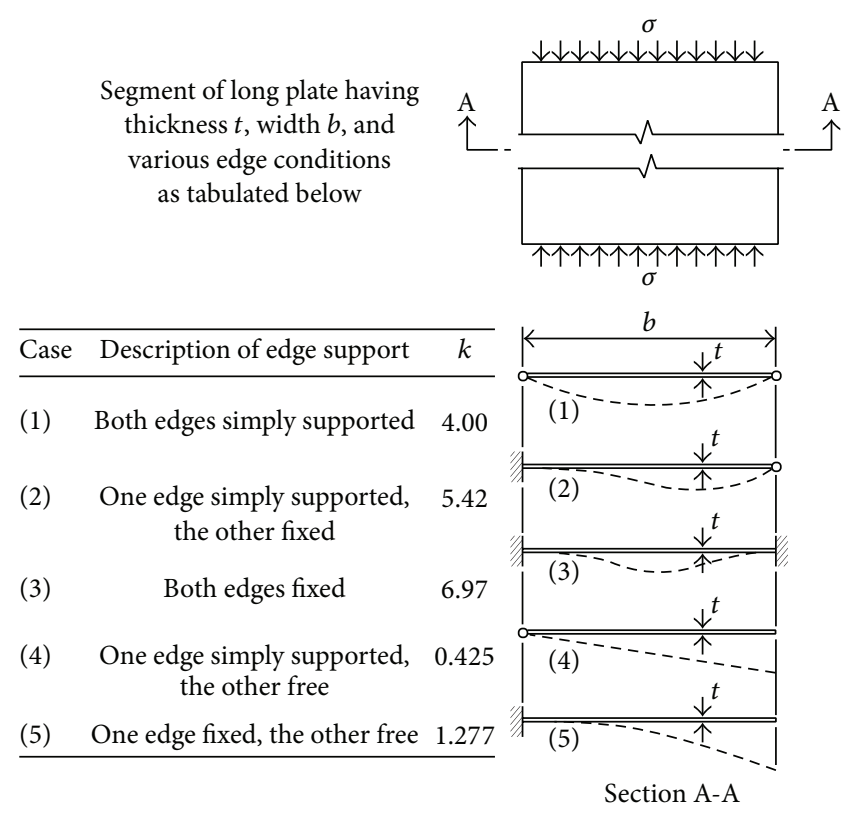

FIGURE 6: Local plate buckling coefficient, $k$ of (3), for plates in compression with varied boundary conditions, Timoshenko et al. [11].

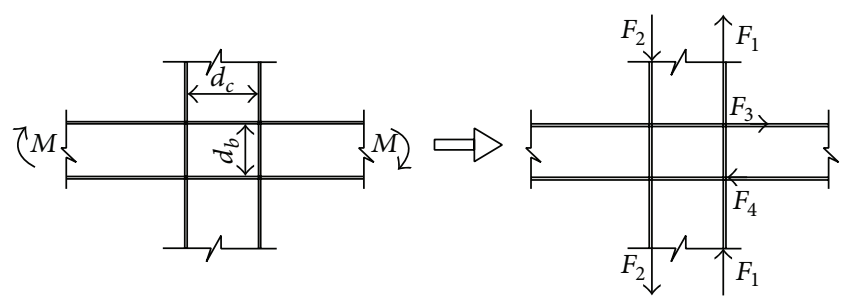

Figure 7: Applied load to the connection plate subjected to cyclic loading.

In the case of combination of compression plus bending stress, the plate can be subjected to in-plane stresses where buckling coefficient, $k$, is dependent on the edge-support conditions and the ratio of bending stress to uniform compression stress. Accordingly, the edges condition and applied load of saddlebag connection are not in good agreement with those of Figure 6 to use proposed buckling coefficient, $k$. The 
TABLE 1: Beam and column properties used in the saddlebag connection.

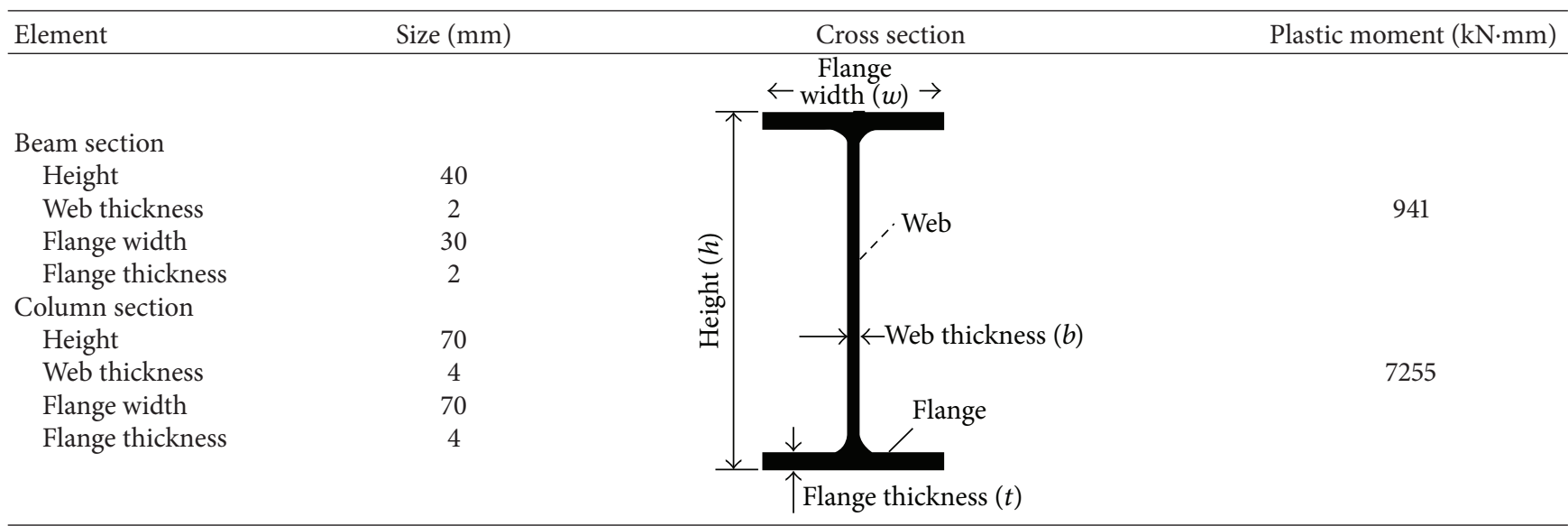

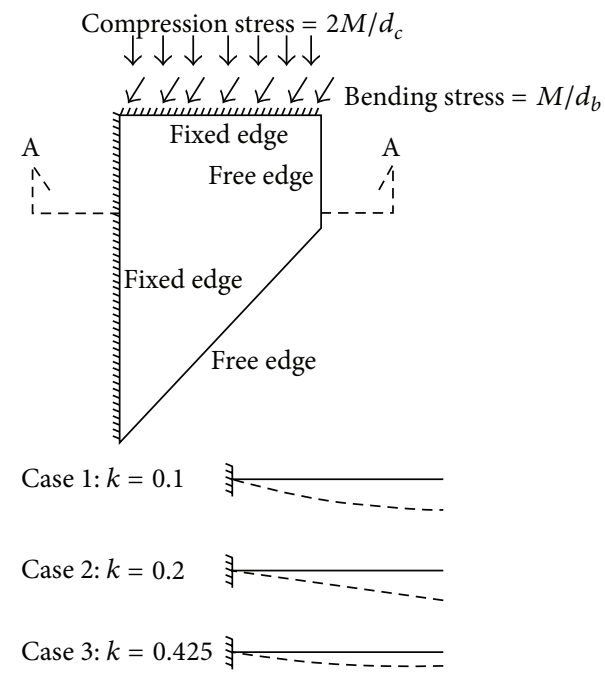

FIGURE 8: Edge conditions of connection plate and proposed buckling coefficients, $k$.

connection plate possesses two fixed supported edges where the other two edges are free. With these particular support conditions, it is not applicable to use buckling coefficient, $k$, provided in Figure 6. Accordingly, in this study three different buckling coefficients, $k$, were considered to address the specific support conditions of saddlebag connection plate. Figure 8 shows the edges condition of saddlebag connection and proposed buckling coefficients.

\section{Numerical Reliability Analysis}

3.1. Case Studies. In this section, the reliability analysis was considered to investigate the suggested formulas and proposed buckling coefficients for connection plate dimension and thickness. For this purpose, the connection plate was designed analytically with suggested formula and then numerically evaluated with ABAQUS software. The details of the new proposed connection for seismic and progressive collapse assessments are shown in Figure 9 and Table 1. All

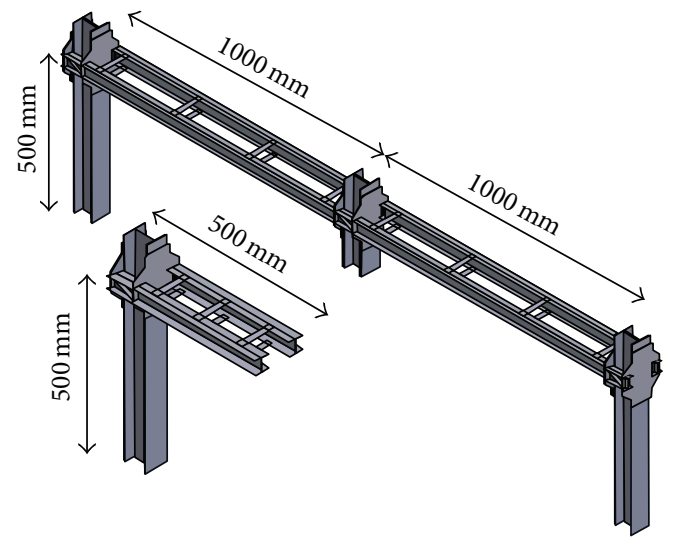

FIGURE 9: Case studies for seismic and progressive collapse assessment.

beams and connections were constructed with built-up I section that have same material properties with connection plate.

The axial load imposed from one of the beams to the connection plate is calculated using (1) as follows:

$$
\begin{aligned}
& F=\frac{M_{p b 1}+M_{p b 2}}{d_{1}} \longrightarrow \\
& F=\frac{941+941}{70}=26.9 \mathrm{kN} .
\end{aligned}
$$

Weld strength per unit length " $R_{w}^{\prime}$ ” with groove weld length of $3.1 \mathrm{~mm}$ for gas metal arc welding as considered in this study was calculated as follows:

$$
\begin{aligned}
& R_{w}^{\prime}=F_{\text {allowable }} \times D_{e} \times \varnothing \longrightarrow \\
& R_{w}^{\prime}=90 \times(0.707 \times 3.1) \times 0.75=148 \mathrm{~N} / \mathrm{mm} .
\end{aligned}
$$

$F_{\text {allowable }}$ is considered as ultimate shear strength of the weld metal (90 MPa for gas metal arc welding "GMAW" subjected to shear). 
The minimum length of $L_{4}$ to transmit the vertical force, $F$, from the groove weld to the column is determined as follows using (2):

$$
\begin{aligned}
& L_{4}=\frac{F}{R_{w}^{\prime}} \longrightarrow \\
& L_{4}=\frac{26.9 \times 1000}{148}=180 \mathrm{~mm} .
\end{aligned}
$$

Considering beam dimension and demanded axial load, the connection plate dimensions based on Figure 5 are summarised as follows:

$$
\begin{aligned}
& L_{1}=\text { Beam flange wide }=30 \mathrm{~mm}, \\
& L_{2}=\text { Column depth }+3 \mathrm{~mm}=80 \mathrm{~mm}, \\
& L_{3}=\text { Beam depth }=40 \mathrm{~mm}, \\
& L_{5}=1.5 \times L_{1}=45 \mathrm{~mm}, \\
& L_{6}=0.4 \times L_{5}=18 \mathrm{~mm} .
\end{aligned}
$$

To define the connection plate thickness, the proposed buckling coefficients, $k$, were considered as follows.

Case $1(k=0.1)$. Consider

$$
\begin{aligned}
\frac{30}{t} & \leq\left\{\frac{0.1 \times \pi^{2} \times\left(2 \times 10^{5}\right)}{12\left(1-0.3^{2}\right)(320 \times 1.33)}\right\}^{1 / 2} \longrightarrow \\
\frac{30}{t} & \leq 6.51 \longrightarrow \\
t & \cong 4.6 \mathrm{~mm} .
\end{aligned}
$$

Case $2(k=0.2)$. Consider

$$
\begin{aligned}
\frac{30}{t} & \leq\left\{\frac{0.2 \times \pi^{2} \times\left(2 \times 10^{5}\right)}{12\left(1-0.3^{2}\right)(320 \times 1.33)}\right\}^{1 / 2} \longrightarrow \\
\frac{30}{t} & \leq 9.21 \longrightarrow \\
t & \cong 3.3 \mathrm{~mm} .
\end{aligned}
$$

Case $3(k=0.425)$. Consider

$$
\begin{aligned}
\frac{30}{t} & \leq\left\{\frac{0.425 \times \pi^{2} \times\left(2 \times 10^{5}\right)}{12\left(1-0.3^{2}\right)(320 \times 1.33)}\right\}^{1 / 2} \longrightarrow \\
\frac{30}{t} & \leq 13.43 \longrightarrow \\
t & \cong 2.3 \mathrm{~mm},
\end{aligned}
$$

where $b$ is equal to $L_{1}$.

3.2. Material Properties. The most commonly accepted method in evaluation of the mechanical properties of metals would be the tension test. Investigation of the engineering

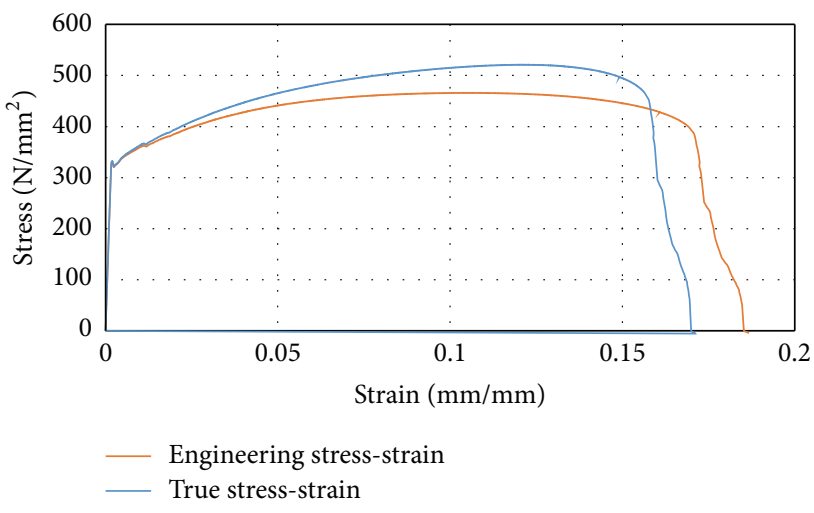

FIGURE 10: Material properties for true stress-strain and engineering stress-strain.

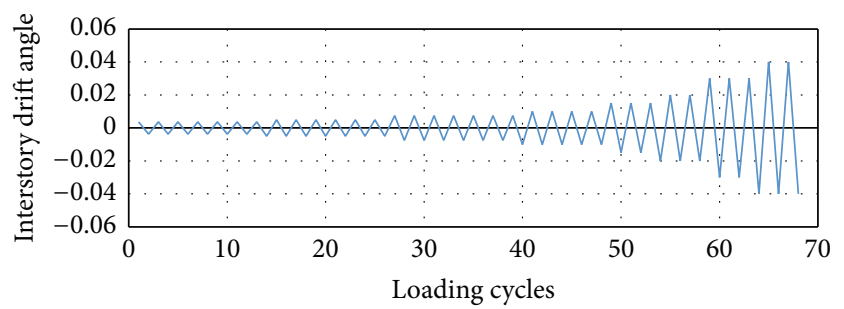

FIGURE 11: The loading protocol used for seismic assessment [12]. Continue loading at increments of $\theta=0.01$ radians, with two cycles of loading at each step.

and true stress-strain relationships of three specimens in conformance with ASTM E8-04 [15] was done in this research. The true and engineering stress-strain curves are presented in Figure 10. After tensile testing, the yield stress and ultimate tensile strength were $320 \mathrm{MPa}$ and $510 \mathrm{MPa}$, respectively.

Determination of true stress-strain for a FEA representation is achieved using the following formulas:

$$
\begin{aligned}
e & =\frac{\Delta l}{l}, \\
S & =\frac{P}{A_{0}}, \\
\sigma & =s(1+e), \\
\varepsilon & =\ln (1+e),
\end{aligned}
$$

where $\sigma$ and $\varepsilon$ are the true stress and strain values and $e$ and $S$ are engineering strain and stress values, respectively (the uniaxial tensile test).

3.3. Loading Protocol and Acceptance Criteria. The 2010 AISC seismic provision [12] loading protocol was considered for seismic evaluation where the protocol specifies a series of load steps and the number of cycles for each as shown in Figure 11. Each load step corresponds to a total interstory drift angle. The load steps were executed and data points recorded at regular intervals. 


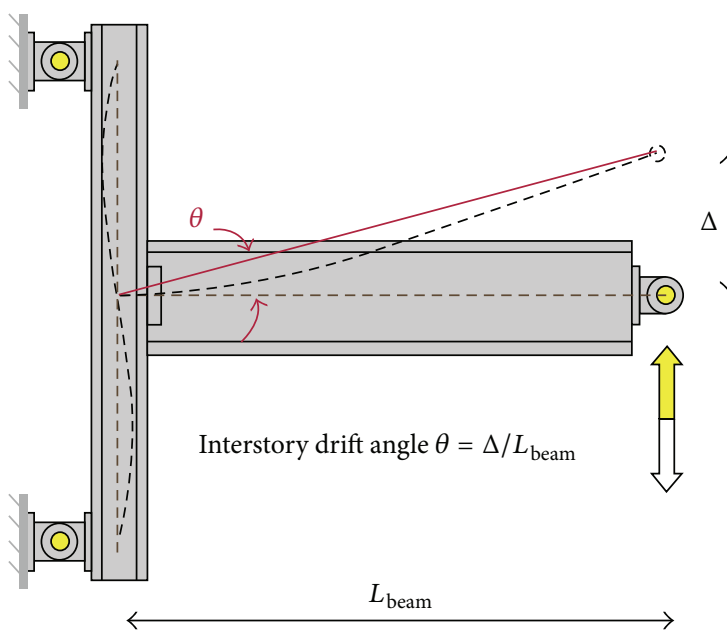

(a)

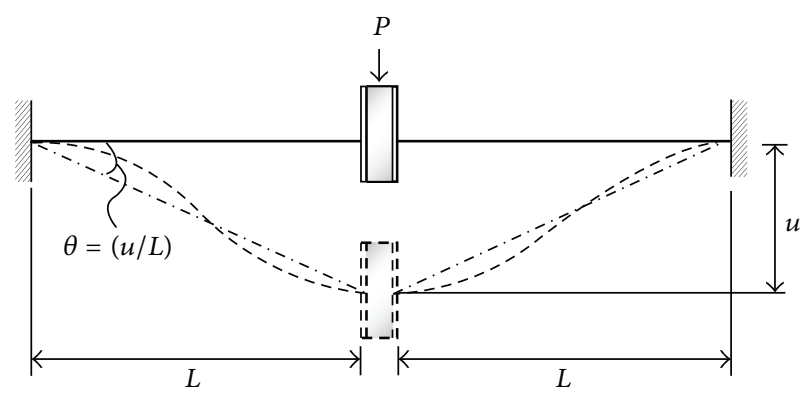

(b)

FIGURE 12: Definition of interstory drift angle (a) and connection rotation capacity (b).

TABLE 2: Acceptance criteria for fully restrained moment connections [13].

\begin{tabular}{lcc}
\hline $\begin{array}{l}\text { Connection } \\
\text { type }\end{array}$ & \multicolumn{2}{c}{ Plastic rotation angle $(\theta)$, radians } \\
\hline Side plate & $\begin{array}{c}\text { Primary element } \\
0.089-1.9^{-5} d\end{array}$ & $\begin{array}{c}\text { Secondary element } \\
0.169-3.9^{-6} d\end{array}$ \\
\hline $\begin{array}{l}\text { Reduced Beam } \\
\text { Section (RBS) }\end{array}$ & $0.05-1.18^{-5} d$ & $0.07-1.18^{-5} d$ \\
\hline
\end{tabular}

d: depth of beam, $\mathrm{mm}$.

The following requirements must be satisfied for beamto-column connections applied for seismic evaluation:

(i) An interstory drift angle of at least 0.04 rad must be sustained by the connection, Figure 12(a).

(ii) The flexural resistance of the connection measured at the column face will be equal to at least $0.80 M_{p}$ of the connected beam at an interstory drift angle of $0.04 \mathrm{rad}$.

For the purpose of progressive collapse assessment, the vertical push-down analysis through gradually increasing the vertical displacement at the location of the removed column was conducted to determine the connection rotational capacity and resistance of the structure against such deformation (Figure 12(b)). The vertical push-down continued until large deformation or collapse mechanism appears at the specimens. Table 2 shows the acceptance criteria based on plastic rotation angle of two different connections in conformity with UFC 4-023 [13] subjected to push-down analysis. For proposed connection, the acceptance criteria based on sideplate moment connection were considered.

\subsection{Finite Element Modelling. The ABAQUS/STANDARD} program was the FE program incorporated in this analysis. It was crucial to consider both the bending behaviour and inplane stresses along with curvature modelling of the deflected shape using the fewest possible elements. Accordingly, the solid C3D8R element was used in the model, having midside nodes (eight-node hexahedral) possessing six degrees of freedom in each node ( 3 displacements and 3 rotations). To simulate the real experimental condition, the coupling technique was considered where this particular technique precludes support and localizes stress concentration. In this technique, one reference point was defined at the applied loading protocol location and tied to the loading zone. Based on tensile test results, the fracture strain was identified and this specific strain (0.16) was defined in the software representative of fracture criteria.

\section{Results and Discussion}

4.1. Progressive Collapse Assessment. To evaluate the adequacy of proposed dimension and buckling coefficients of saddlebag connection plate subjected to push-down analysis, the numerical investigation was carried out in this section.

In Case 1, the von Mises stress distribution clearly indicated that saddlebag connection plate develops the full capacity, $M_{p}$, of connected beam without any buckling or stress concentration. The column shear panel zone also experienced negligible stress at the final stage compared to the connected beams. Generally, the proposed buckling coefficient resulted in conservative thickness for connection plate as it experienced much lesser stress compared to beam. Figure 13(a) depicts the failure mode and behaviour of connection plate at the final stage of progressive collapse analysis.

The connection plate designed in accordance with buckling coefficient (Case 2) also showed adequate performance to resist progressive collapse where it develops the full capacity of connected beam without buckling or stress concentration, Figure 13(b). Besides, von Mises stress distribution revealed that connection plate experienced stress close to yield stress. This issue emphasises that proposed buckling coefficient of 


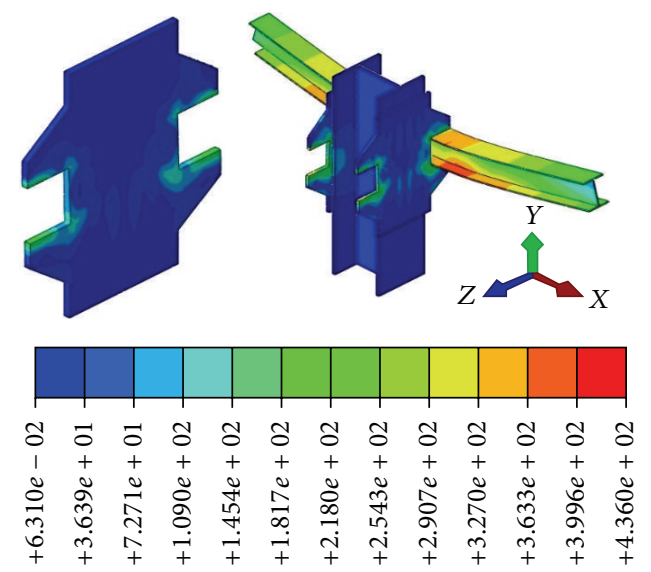

$S$, Mises (avg: 75\%)

(a)
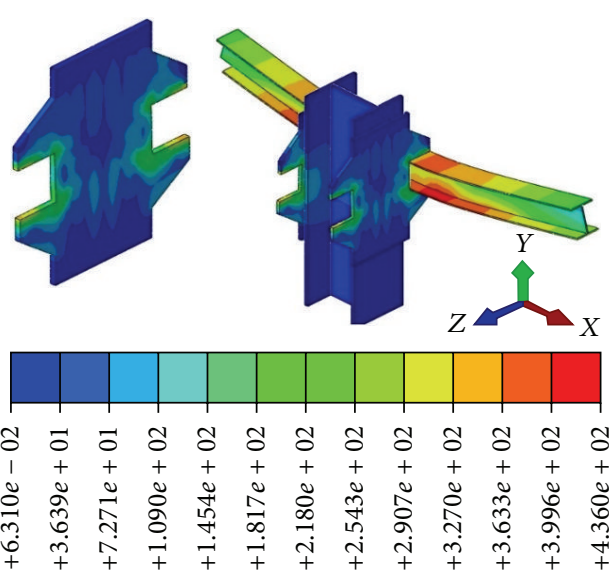

S, Mises (avg: 75\%)

(b)
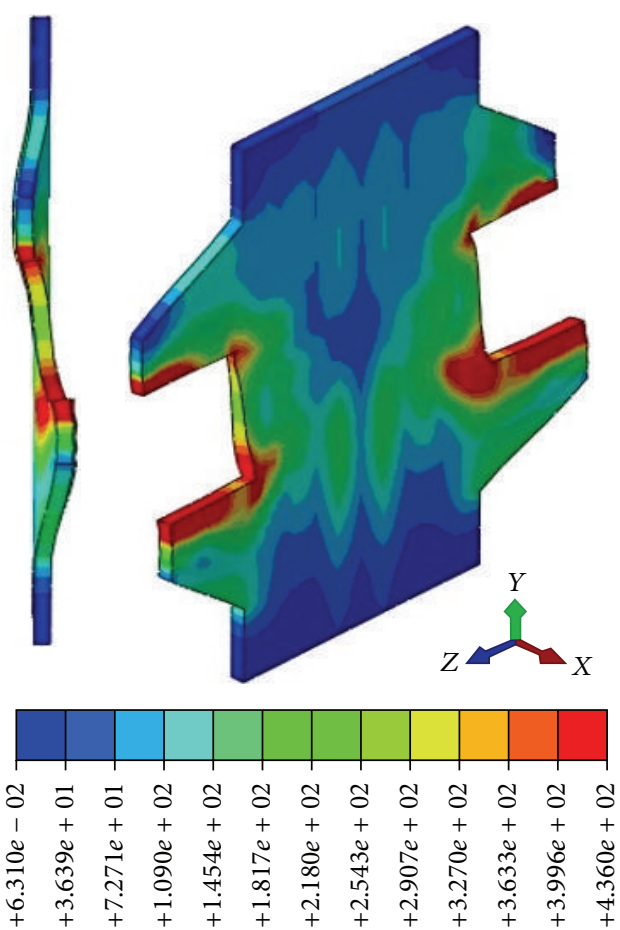

S, Mises (avg: 75\%)

(c)

FIGURE 13: Saddlebag connection behaviour at the end of progressive collapse test.

Case 2 addressed progressive collapse design requirements as well as economic aspects.

Using buckling coefficient of Case 3 to calculate the connection plate thickness resulted in unconservative results where connection plate experienced buckling before developing the full capacity of connected beams. This issue is against progressive collapse design philosophy that demands adequate plastic hinge rotation angle of beam to column connection subjected to sudden column removal. As shown in Figure 13(c), connection plate designed with buckling coefficient Case 3 failed to develop plastic hinges rotation angle.
The numerical plots of vertical load $(\mathrm{KN})$ versus the plastic hinge rotation angle (radians) for buckling coefficient of Case 3 are shown in Figure 14. According to this figure, connection plate designed with buckling coefficient of Case 2 successfully developed the plastic hinges rotation angle around 0.22 radians that only took place in connected beams.

4.2. Seismic Assessment. To evaluate the adequacy of proposed dimension and buckling coefficients of saddlebag connection plate subjected to cyclic loading, the numerical investigation was carried out in this section. 


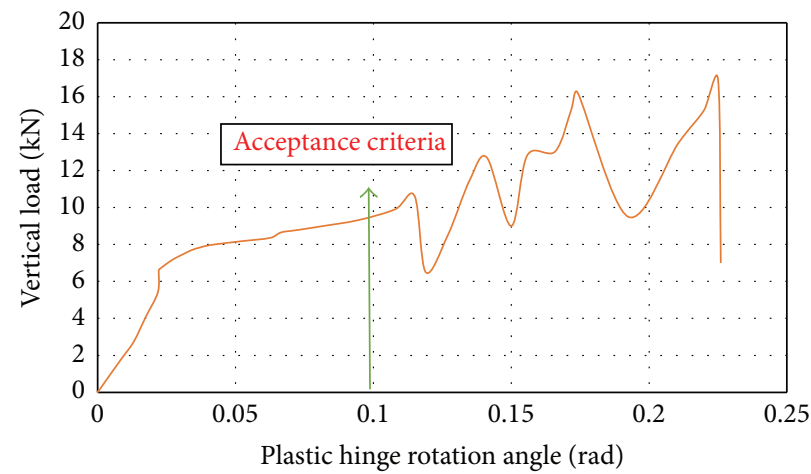

FIGURE 14: Vertical load versus plastic hinge rotation angle for progressive collapse tests.

The result explicitly declares that, by using buckling coefficients of Cases 1 and 2, connected beams experienced plastic moment, $M_{p}$, where connection plate remains in elastic form at the end of cyclic loading (0.06-radian interstory drift angle). Besides, the connection provides adequate energy dissipation capacity which is considered as important characteristic to resist cyclic loading. However, similar to progressive collapse assessment, the result revealed that buckling coefficients of Case 1 lead to conservative thickness where it experienced much lesser stress compared to yield stress as shown in Figure 15(a). Numerical results showed that using buckling coefficient of Case 2 lead to reasonable stress distribution among connection plate and connected beams where connection plate experienced stress close to yield stress. However, the failure mode at the final stage of cyclic loading revealed that connection plate did not experience buckling or stress concentration, Figure 15(b).

Figure 15(c) shows that connection plate experienced buckling before developing the full capacity of connected beams by using buckling coefficient of Case 3. This issue makes the proposed connection vulnerable to cyclic loading. Accordingly, the proposed buckling coefficient of Case 3 lead to unconservative results for connection plate which could not protect beam to column connection against seismic loading.

The computed moment at the column face versus interstory drift angle recognized as the global seismic response of the saddlebag connection specimen is shown in Figure 16. The saddlebag connection resists at least one cycle of 0.06 radian interstory drift angle before reaching the failure strain criteria (0.16). Accordingly, the saddlebag connection addressed the minimum requirement (0.04-radian interstory drift angle) of 2010 AISC seismic provision.

In a structural system deforming beyond its elastic limit, the energy imparted to the structure is dissipated by damping and yielding of the elements. Most structures subjected to large earthquake motions must be able to deform well beyond the yield deformation limit. The total energy dissipated by the specimens was determined as the area enclosed by the load versus displacement hysteresis loop for each cycle (Figure 17). The amount of energy dissipated by each specimen depended on the applied lateral load, as well as on the shape of the load versus displacement hysteresis loops. Figure 18 shows the loops of energy dissipated per each cycle for proposed saddlebag connection. As can be seen in this figure, the fuller hysteretic loops have resulted in proposed saddlebag connection subjected to cyclic loading. The fuller hysteresis loops of saddlebag connection indicate its adequacy in seismic energy removal and ductility capacity which imply better performance when comparing with other connection with similar strength. Besides, stable hysteretic loops with large energy dissipation capacity of saddlebag connection are considered as a guarantee for a better deformation performance to address inelastic deformation demands of earthquake.

\section{Summary of Findings and Concluding Remarks}

The seismic and progressive collapse performance of a saddlebag connection was investigated in this paper. The interstory drift angle and flexural strength in conformance with AISC Seismic Provisions (2010) along with plastic rotation angle based on UFC 4-023-03 (2010) guideline were the major acceptance criteria for seismic and progressive collapse evaluation, respectively. The following conclusions have been made regarding the experimental and numerical results:

(i) The saddlebag connection was able to achieve adequate rotational capacity, develop catenary action, and also develop the full inelastic capacity of the connecting beam. Therefore, it could be a robust and effective solution in case of a terrorist bomb blast, including progressive collapse.

(ii) Considering the seismic performance results, one complete cycle of an interstory drift angle of $0.06 \mathrm{rad}$ was satisfied by the saddlebag connection. Hence, this connection can be used in SMF according to 2010 AISC Seismic Provisions.

(iii) The comprehensive hysteretic loops have resulted in saddlebag connection subjected to cyclic loading which indicate its adequacy to dissipate seismic loading. 

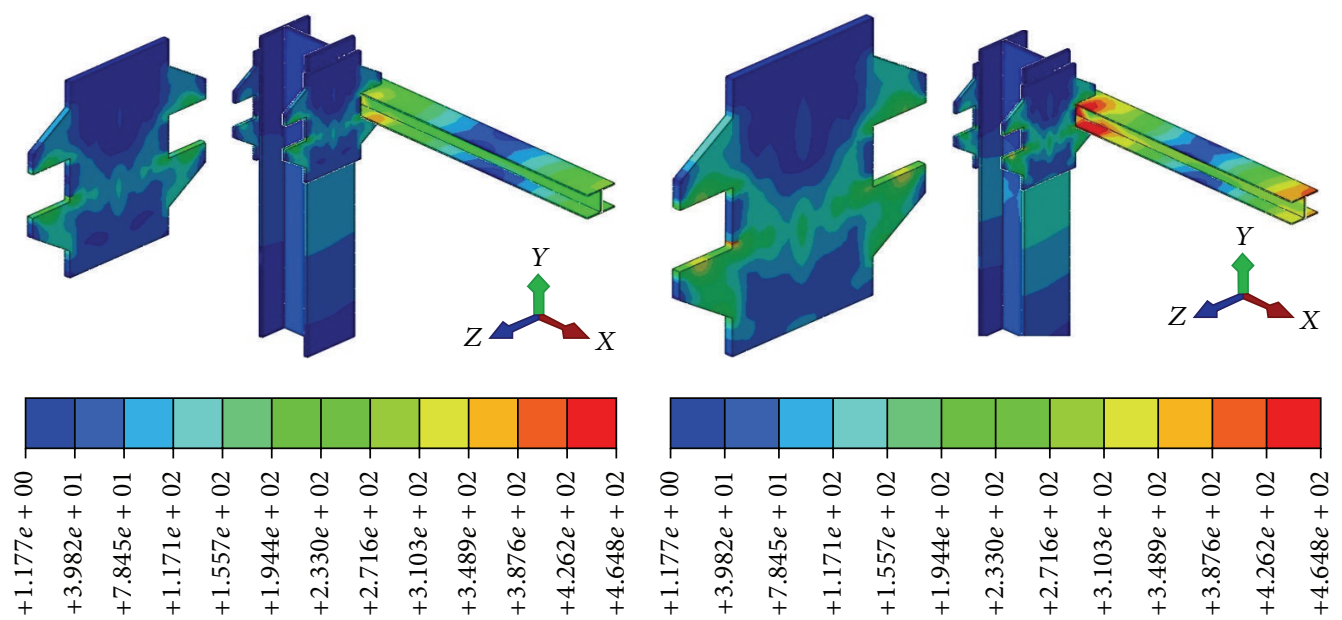

S, Mises (avg: $75 \%$ )

$S$, Mises (avg: $75 \%$ )

(a)

(b)
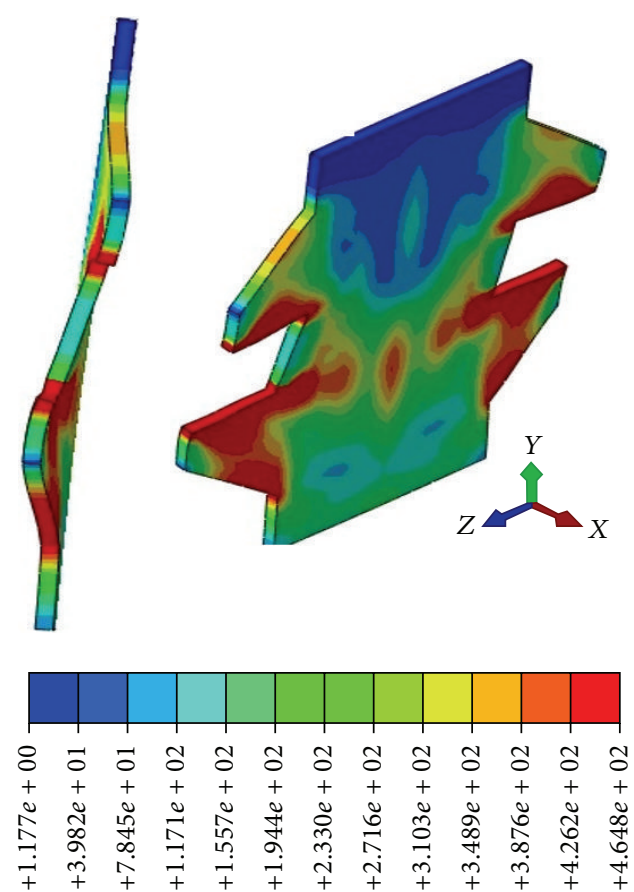

S, Mises (avg: 75\%)

(c)

FIgURE 15: Saddlebag connection behaviour at the end of cyclic test.

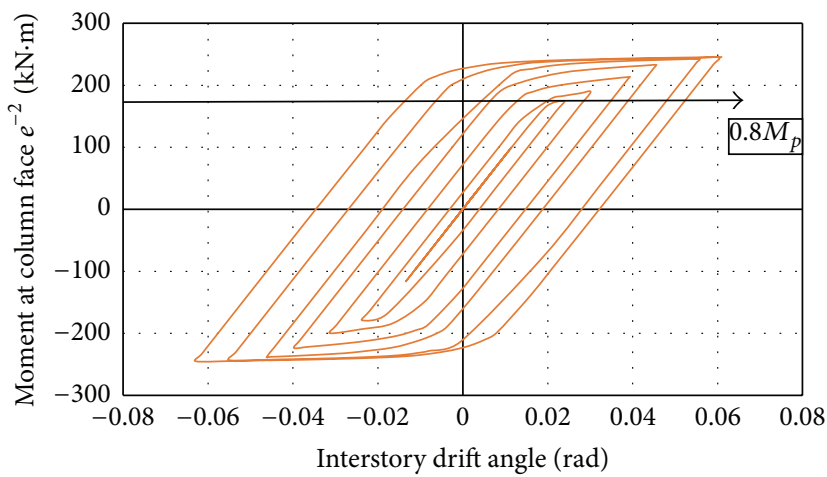

Figure 16: Moment at column face versus interstory drift angle. 


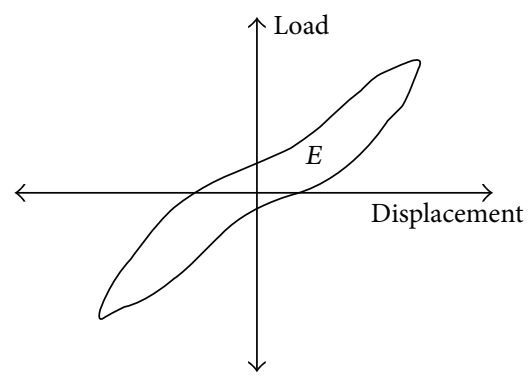

FIGURE 17: Energy dissipated per cycle definition.

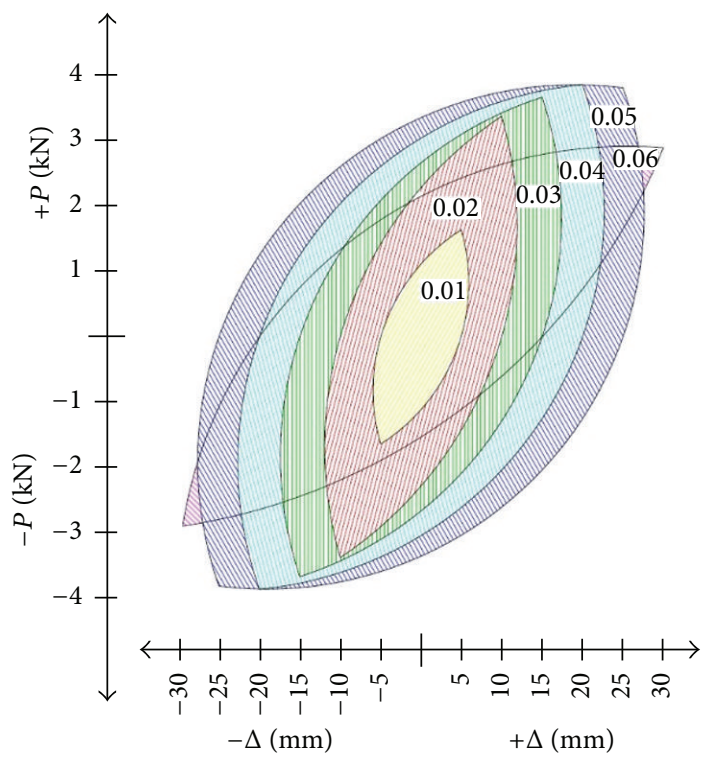

FIGURE 18: Calculation of energy dissipated by saddlebag connection.

\section{Conflict of Interests}

The authors declare that there is no conflict of interests regarding the publication of this paper.

\section{Acknowledgments}

The authors wish to thank the esteemed technical staff of the Universiti Teknologi Malaysia (UTM) for their cooperation and support in this study. The financial support (Research Grant Q.J130000.2422.02G84) provided by the university for conducting the experimental work is also admirable.

\section{References}

[1] S.-J. Chen, C. H. Yeh, and J. M. Chu, "Ductile steel beam-tocolumn connections for seismic resistance," Journal of Structural Engineering, vol. 122, no. 11, pp. 1292-1299, 1996.

[2] J. Zhang and P. Dong, "Residual stresses in welded moment frames and implications for structural performance," Journal of Structural Engineering, vol. 126, no. 3, pp. 306-315, 2000.

[3] C.-M. Uang, Q.-S. Yu Kent, S. Noel, and J. Gross, "Cyclic testing of steel moment connections rehabilitated with RBS or welded haunch," Journal of Structural Engineering, vol. 126, no. 1, pp. 5768,2000 .

[4] M. D. Engelhardt and A. S. Husain, "Cyclic-loading performance of welded flange-bolted web connections," Journal of Structural Engineering, vol. 119, no. 12, pp. 3537-3550, 1993.

[5] D. L. Houghton, "Steel moment resisting frame beam-tocolumn connections," Google Patents, 1997.

[6] S. Marjanishvili and E. Agnew, "Comparison of various procedures for progressive collapse analysis," Journal of Performance of Constructed Facilities, vol. 20, no. 4, Article ID 010604QCF, pp. 365-374, 2006.

[7] J. E. Karns, D. L. Houghton, B. E. Hall, J. Kim, and K. Lee, "Blast testing of steel frame assemblies to assess the implications of connection behavior on progressive collapse," in Proceedings of the ASCE Structures Congress, St. Louis, Mo, USA, May 2006.

[8] A. S. Usmani, Y. C. Chung, and J. L. Torero, "How did the WTC towers collapse: a new theory," Fire Safety Journal, vol. 38, no. 6, pp. 501-533, 2003.

[9] B. Yang and K. H. Tan, "Numerical analyses of steel beamcolumn joints subjected to catenary action," Journal of Constructional Steel Research, vol. 70, pp. 1-11, 2012.

[10] G. Xu and B. R. Ellingwood, "Disproportionate collapse performance of partially restrained steel frames with bolted T-stub connections," Engineering Structures, vol. 33, no. 1, pp. 32-43, 2011.

[11] S. P. Timoshenko, J. M. Gere, and W. Prager, "Theory of elastic stability," Journal of Applied Mechanics, vol. 29, p. 220, 1962.

[12] American Institute of Steel Construction, Prequalified Connections for Special and Intermediate Steel Moment Frames for Seismic Applications, AISC Connection Prequalification Review Panel, 2010.

[13] Department of Defense, "Design of buildings to resist progressive collapse," UFC 4-023-03, Department of Defense, 2010.

[14] AISC, Plastic Design Specifications for Structural Steel Buildings, American Institute of Steel Construction, Chicago, Ill, USA, 1989.

[15] ASTM, "Standard test methods for tension testing of metallic materials," ASTM Standard E8-04, 2004, Annual Book of ASTM Standards, Volume 3. 


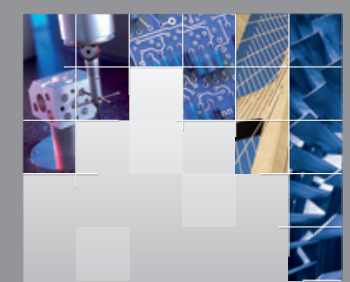

\section{Enfincering}
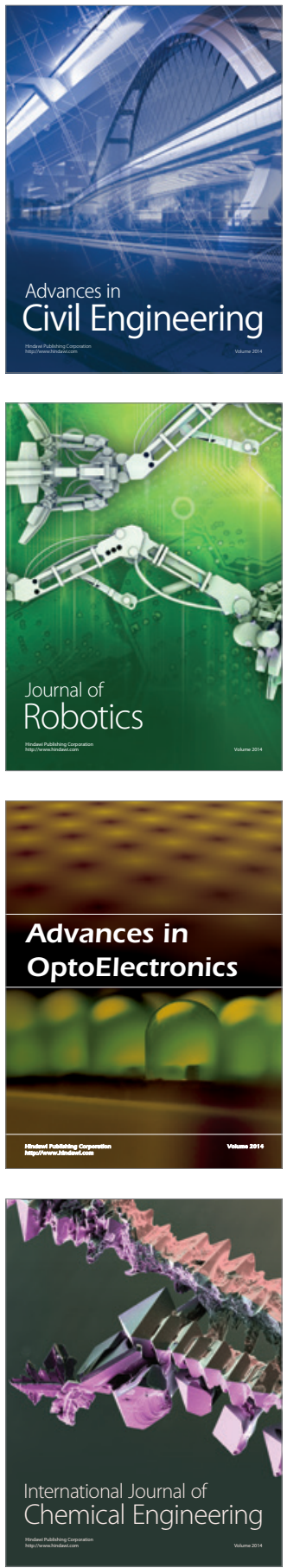

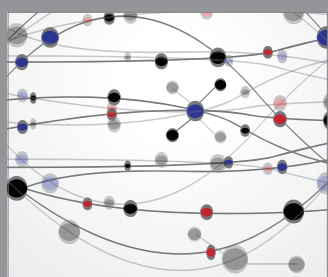

The Scientific World Journal

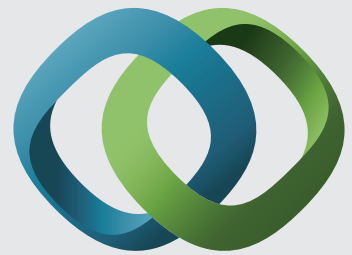

\section{Hindawi}

Submit your manuscripts at

http://www.hindawi.com
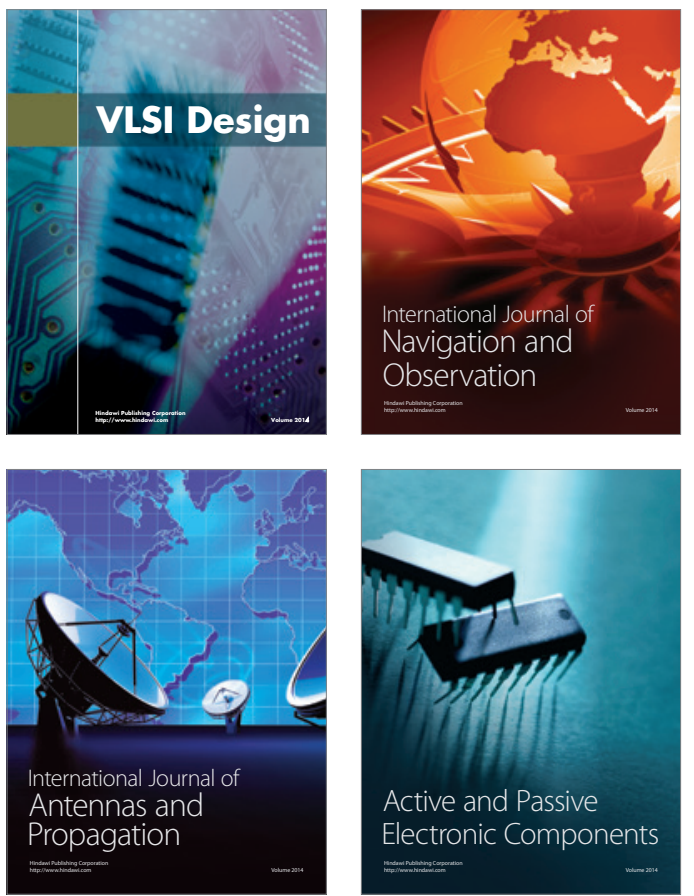
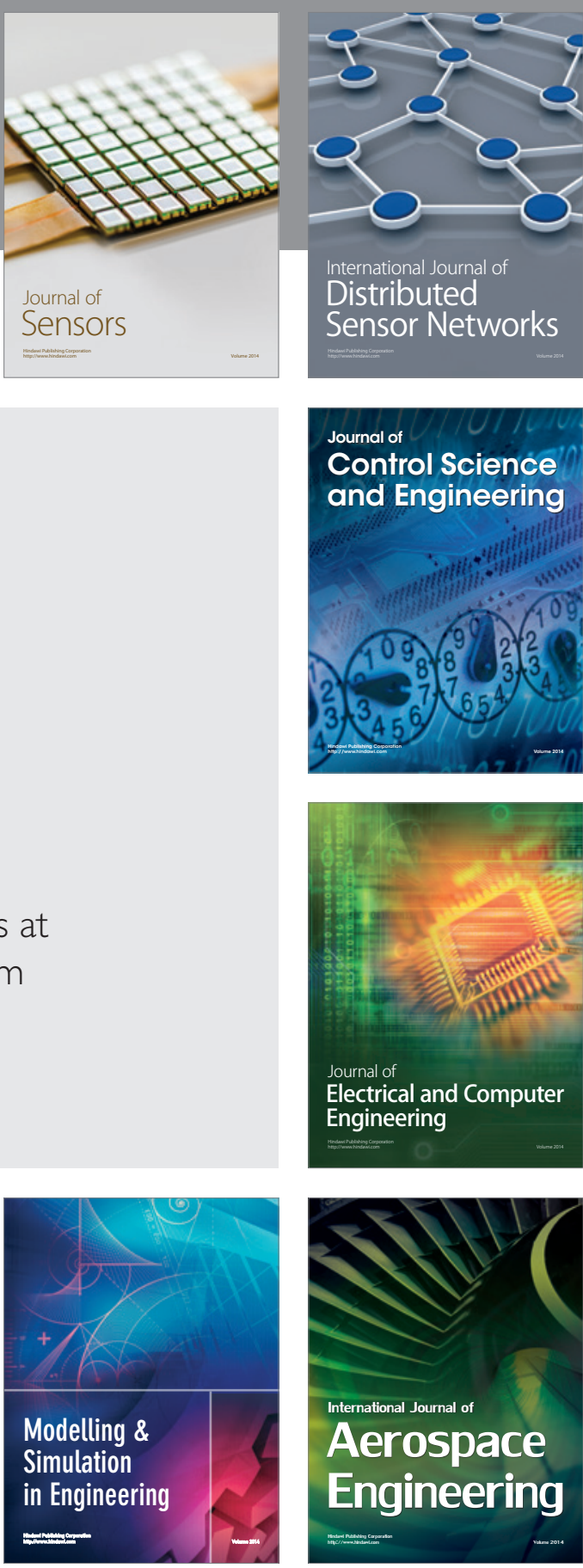

International Journal of

Distributed

Sensor Networks

Journal of

Control Science

and Engineering
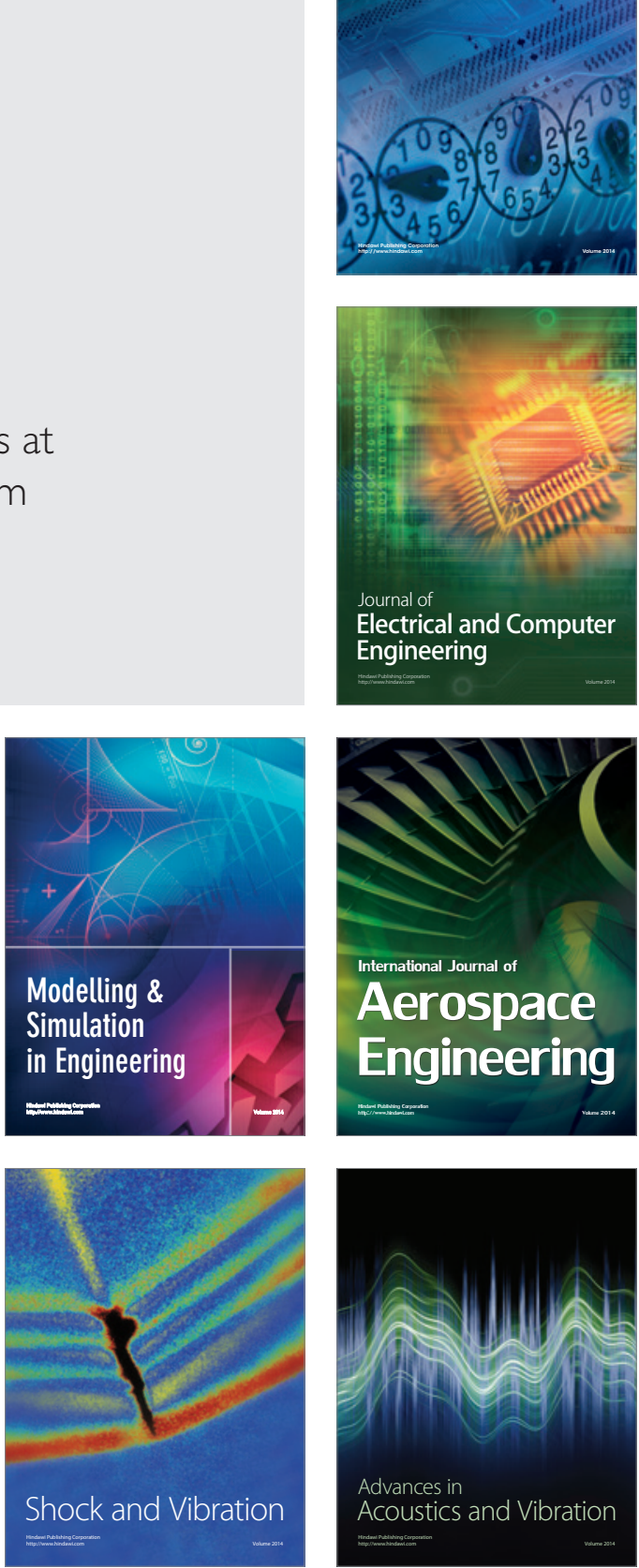